\title{
Téoros
}

Revue de recherche en tourisme

\section{Activités et foyers touristiques en Méditerranée : scénario pour un proche futur}

\section{Jean-Pierre Lozato-Giotart}

Volume 12, numéro 2, juillet 1993

La prévision-prospective du tourisme

URI : https://id.erudit.org/iderudit/1078024ar

DOI : https://doi.org/10.7202/1078024ar

Aller au sommaire du numéro

Éditeur(s)

Université du Québec à Montréal

ISSN

0712-8657 (imprimé)

1923-2705 (numérique)

Découvrir la revue

Citer cet article

Lozato-Giotart, J.-P. (1993). Activités et foyers touristiques en Méditerranée :

scénario pour un proche futur. Téoros, 12(2), 35-39.

https://doi.org/10.7202/1078024ar d'utilisation que vous pouvez consulter en ligne.

https://apropos.erudit.org/fr/usagers/politique-dutilisation/ 


\section{Activités et foyers touristiques en Méditerranée : scénario pour un proche futur

\author{
Jean-Pierre Lozato-Giotart"
}

Demain ne sera pas comme bier, il sera nouveau et dépendra de nous. Gaston Berger

\section{Une problématique aux limites incertaines}

Les difficultés de la prospective touristique

Il existe au moins deux types de difficultés auxquelles se heurte toute démarche prospective touchant au futur du tourisme, en Méditerranée comme ailleurs dans le monde.

La première difficulté tient à l'objectif même de la prospective qui est, a priori, de définir les scénarios de l'avenir touristique méditerranéen. Peut-on vraiment prévoir, avec plus ou moins d'exactitude, ce que seront les futures fréquentations touristiques dans l'ensemble du bassin méditerranéen? Et à quelle échéance dans le temps est-il possible d'apporter des réponses probables à l'évolution touristique du monde méditerranéen? À court ou à long terme, à l'horizon 2000, comme le firent $\mathrm{H}$. Kahn et A.J. Wiener du Hudson Institute ${ }^{(2)}$ ou au-delà, comme le firent les experts du Plan Bleu? ${ }^{\text {() }}$

La deuxième difficulté, dont dépend finalement toute l'analyse prospective, est d'ordre méthodologique: sur quels indicateurs et selon quelles méthodes d'analyse peut-on proposer des scénarios annonçant les futures réalités touristiques en Méditerranée? Ilestclair quele choix de tel ou tel paramètre peutengendrer des prévisions tout à fait contradictoires, sans que l'on puisse pour autant condamner la démarche prospective. Prévoir le futur touristique méditerranéen n'a pas toujours été facile si l'on compare les *erreurs* prévisionnelles passées. Qui avait vraiment prévu les chutes de fréquentation

Monsieur Jean-Pierre Lozato-Giotart est professeur au Magistere de Relations Internationales et d'Action â l'étranger (responsable de l'option Tourisme International) a I"UFR Espaces-Cultures, Université de Nice Sophia-Antipolis internationale à destination de la Méditerranée enregistrées en 1980, 1986, 1989 et 1991 (figure 1)? On serait tenté, une fois encore, de dire que les experts en futurologie socio-économique se trompent toujours. Pareil jugement excessif ne doit pas neutraliser l'ambition prospective qui consiste à rechercher dans le présent ce qui peut permettre de préparer l'avenir.

\section{La problématique spatiale : intérêt et limite}

Le tourisme, et d'une façon plus large l'ensemble des loisirs, met en jeu de nombreux paramètres - sociaux, économiques, juridiques, techniques, géographiques, politiques - qui finissent par interférer au sein d'un espace réceptif commun (figure 2). En Méditerranée, la problểmatique spatiale est riche de la complexité des liens qui unissent les terres et les mers. Le rôle deshommes dans l'organisation touristique de l'espace méditerranéen demeure fondamental, d'où l'importance des facteurs socio-économiques et socio-culturels dans toute analyse prospective du tourisme en Méditerranée à l'aube du XXI ${ }^{e}$ siècle.

Il n'est question ici que d'une tentative prospective limitée à l'étude de l'évolution future des principaux flux émetteurs et des principanux foyers récepteurs touristiques méditerranéens. Le principal intếrêt d'une démarche spatiale étant de proposer une méthodologie capable d'aider à la compréhension plus générale des possibles scénarios touristiquesen Méditerranée. Le poids des facteurs géographiques - d'après les données naturelles jusqu'aux données géopolitiques - sur les activités touristiques ne doit pas masquer la difficulté à en délimiter les effets si l'on ne tient pas compte des autres paramètres. Cependant, que ce soit de façon positive ou de façon négative, l'impact du tourisme affecte très directement les zones réceptrices et les enjeux territoriaux qui peuvent en découler pour les sociétés d'accueil méditerranéennes concernées.

\section{Une fréquentation touristique croissante mais inégale}

\section{Le premier *lac* \\ touristique mondial}

En 1989 , les pays riverains de la Méditerranée ont accueilli plus de 145 millions de touristes internationaux, soit près de $32 \%$ du nombre mondial de touristes internationaux $x^{(4)}$. Depuis 1970, d'après l'Organisation mondiale du tourisme, la Méditerranée a connu une croissance moyenne annuelle supérieure à $5 \%$. Si l'on tient comptedu fort développement du tourisme national dans presque tous les pays riverains, la Méditerranée s'impose encore aujourd'hui commele premier *lacs ou foyer récepteur touristique mondial.

Pourtant, depuis 1990, la croissance du nombre de touristes internationaux à destination de la Méditerranée est en netrecul (entre $2 \%$ et $3 \%$, soit deux fois moins qu'avant). Cela mérite d'être d'autant plus signalé que la fréquentation touristique mondiale n'a pas connu de décélération comparable à celle enregistrée en Méditerranée. Que faut-il en conclure? Est-ce un épisode conjoncturel ou, au contraire, l'amorce d'une évolution durable?

\section{Une évolution imprévue}

En se référant aux diverses démarches prospectives antérieures, comme celles de A. Edwards $s^{(5)}$, on peut constater qu'aucune n'avait prévue les récentes fluctuations de la fréquentation touristique internationale dans le bassin méditerranéen. Tous les plans de développement touristique de la plupart des pays méditerranéens étaient orientés vers la croissance de la fréquentation, en particulier dans les plus importants pays récepteurs nord-africains tels que la Tunisie et le Maroc ${ }^{(0)}$. La mauvaise surprise fut donc d'autant plus brutale que la *fuiteo de la clientèle a été rapide et massive. En 1991, monsieur Abdou Belbekir, président de l'Association des professionnels du tourisme de Marrakech, déclarait: 
*Nous avons réellement l'impression que le pays va devoir repartir à zéros.7).

Cette opinion pessimiste traduisait le désarroi des responsables marocains devant la plus forte crise touristique touchant leur pays ( $60 \%$ des touristes étrangersen moins et des hôtels presque vides). L'effondrement des destinations yougoslaves, et par ricochet de la crise balkanique, le freinage enregistré en Grèce, à quoi se sont ajoutés l'éclatement politique de la Bulgarie et de la Roumanie ainsi que les troubles intérieurs algériens, furent autant de facteurs d'insécurité interrompant l'augmentation croissante de la fréquentation touristique en Méditerranée. Si le conflit israëlo-arabe est assez ancien pour ne plus guère influencer trop directement les prévisions, personne, ou presque, n'avaitenvisagéledouble choc de la guerre du Golfe (août 1991février 1992) et des conflits yougoslavessur la fréquentation touristique en Méditerranée. Les troubles géopolitiques récents ont eu des effets négatifs immédiats - car l'insécurité est incompatible avec le tourisme - et plus durables, semble-t-il, que les seules prospectives à caractère socioéconomique.

\section{Redéploiement ou repli?}

L'observation du trenddu trafic touristique à destination de la Méditerranée de 1970 à 1992 laisse apparaître une croissance annuelle moyenne comprise entre $4,5 \%$ et 5 $\%$ selon les sources statistiques. Les situations extrêmes ont été enregistrées en $1972(+11,7 \%)$ et $1974(-6,2 \%)$. En fait, jusqu'en 1973, la fréquentation touristique internationale a connu une croissance quasi ininterrompue en Méditerranée jusqu'à *l'accident de 1974 dô aux conséquences de la première grande crise pétrolière mondiale. Depuis, la fréquentation touristique internationale à destination de la Méditerranée a été nettement plus irrégulière avec des années fortes $(+9,2 \%$ en $1975,+8,8 \%$ en 1988$)$ et des années faibles $(+0,6 \%$ en $1976,+0,7 \%$ en $1980,+1,1 \%$ en 1986 ou $+1 \%$ en 1991 ). Il est tout de même remarquable qu'au cours de la période 1975-1992, une année sur deux a connu soit un freinage ou un recul de la fréquentation touristique internationale pour l'ensemble du bassin méditerranéen. Les soubresauts monétaires et financiers (crise de 1979-1980, récession de 1983) et géopolitiques (attentats de 1986, guerre du Golfe en 1991 et conflits yougoslaves en
FIGURE 1

Arrivées et recettes touristiques

( $\mathrm{M}=$ Méditerranée)
ARRIVÉES
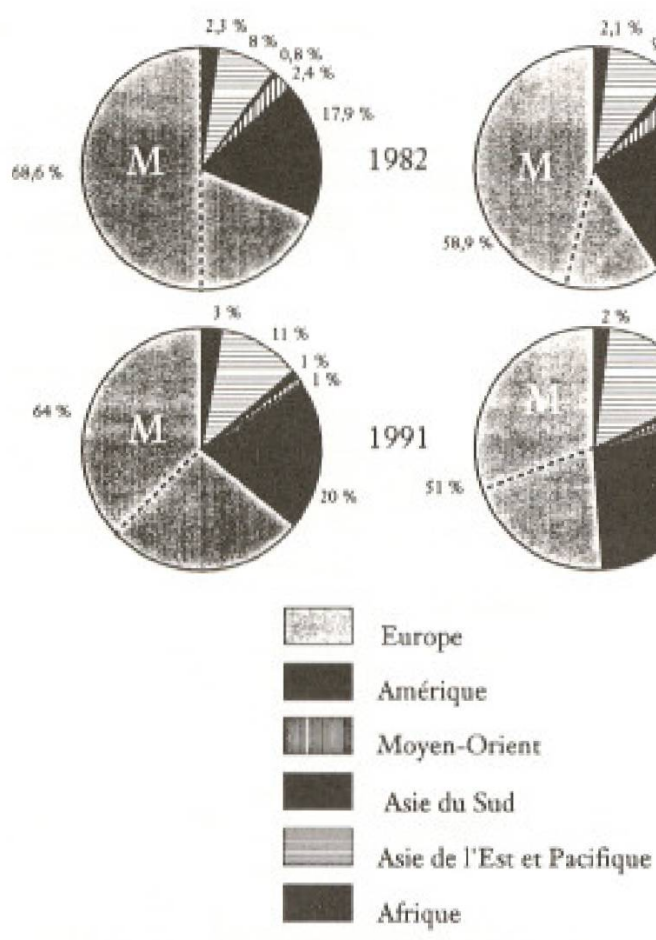

RECETTES

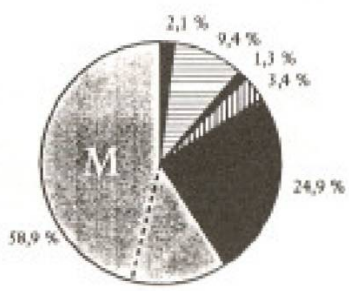

1991

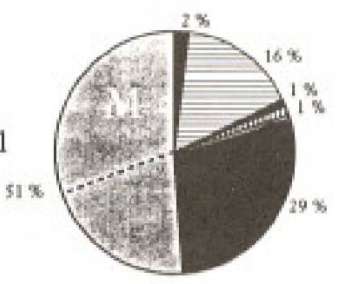

1992) ont affecté très directement certains marchés touristiques méditerranéens. On est en droit de se demander s'il s'agit d'une situation durable ou conjoncturelle.

On peut penser que, à brève échéance, la fréquentation touristiqueen Méditerranée ne retrouvera pas sa forte croissance passée. Cela à cause au moins de deux facteurs essentiels:

- le premier facteur tient à la commune de plus en plus vive des autres grands foyers récepteurs internationaux, notamment ceux de l'Asie du Sud-Est et de la zone caraíbe. Dès les années 1970, des enquêtes menées sur la Côte

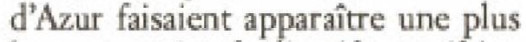
longue rotation de clientèle, aussi bien nationale qu'internationale, à destination des rivieras franco-italiennes ${ }^{(\%)}$;

- ledeuxième facteur estla conséquence directe de l'extension, plus ou moins durable, de l'instabilité politique et donc de l'insécurité dans des pays méditerranéens (ex-Yougoslavie, Algérie, Liban). Les zones deconflits, même périphériques (golfe persique) et la montée d'un islam intégriste ont entraîné des fortes baisses de fréquentation touristique internationale de la Méditerranée orientale jusqu'au Maroc, de même qu'en Grèce à cause des guerres civiles opposantles Serbes aux Croates et aux Bosniaques.

L'insécurité est donc devenu le principal facteur d'affaiblissement de la fréquentation touristique en Méditerranée. A l'inverse, cela est corroboté par la croissance forte du tourisme en Turquie ( $+40 \%$ en 1992!) et en Méditerranée nord-occidentale apparaissant aujourd'hui comme des zones d'accueil sûres aux yeux de la clientèle internationale issue des pays riches. Par ailleurs, il est remarquable que les facteurs économiques, et plus particulièrement monétaires, n'ont déclenché d'aussi brutales variations de la fréquentation touristique internationale en Méditerranée.

Malgré la crise de fréquentation enregistréedans les zones méditerranéennesjugées les minoins sûres, on ne peut pas encore conclure à un repli du tourisme en 


\section{FIGURE 2}

Répartition géographique des villages du Club Méditerrannée (1992) et problèmes gćopolitiques

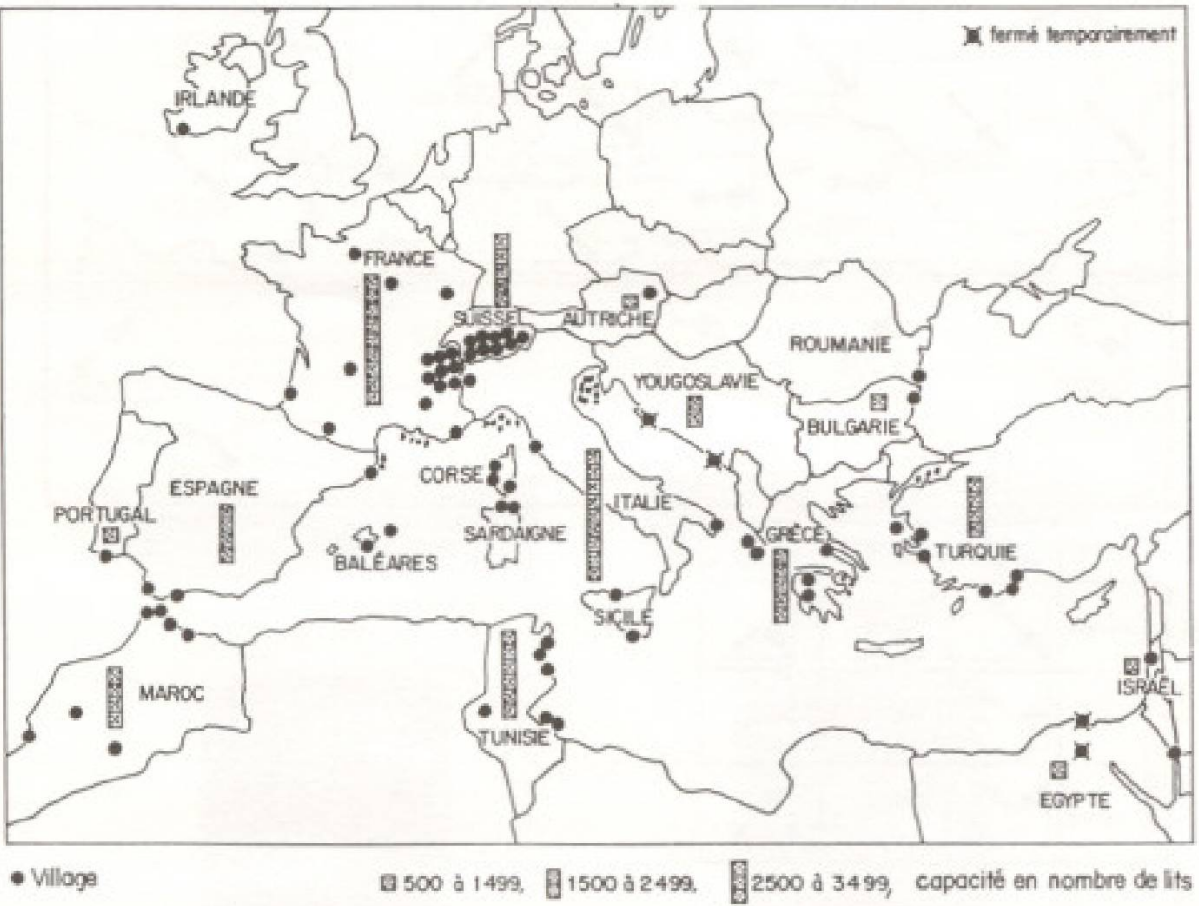

Méditerranée. On assiste plutôt à un redéploiement sous deux formes:

- enpremier lieu, la majoritédes touristesayant déserté les zones d'insécurité s'est redéployée sur les rivages de l'Europe méditerranéenne et en Turquie. Une partie semble, toutefois, avoir déserté momentanément la Méditerranée pour d'autres horizons (Amérique du Nord et Caraibes);

- endeuxième lieu, l'évolution du nombre de nuitées et de la durée moyenne de séjour, en baisse depuis 1987, laisse entrevoir l'amorce d'un redéploiement durable du temps de vacances et de loisirs (figure 3 ).

Ce phénomène est imputable au raccourcissement des vacances d'été au profit des congés de plus courte durée (de 2 à 6 jours) qu'accentuent, sans doute, les problèmes socio-économiques que connaissent de nombreux foyers émetteurs européens et nord-américains.

Ainsi, l'analyse de l'évolution récente de la fréquentation touristique en Méditerranée soulèveau moinsle problèmed'une tendance lourde au redéploiement probable du tourisme méditerranéen à l'horizon 2000. Dès lors, on peut essayer d'esquisser les scénarios possibles et leurs conséquences géographiques.

\section{Scénario et prospective spatiale}

\section{Le scénario pessimiste}

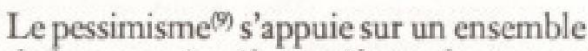
de facteurs négatifs parmi lesquelsont peut distinguer:

- l'accentuation des troubles et conflits politiques s"étendent au Kosovo et à l'Albanie, à la Bulgarie et à la Roumanie, à l'ensemble des pays maghrébins, à l'Eggypte et au ProcheOrient;

- une orise sociale et économique profonde entraînant une baisse des revenus des touristes étrangers européens, nordaméricains et japonais;

- une dégradation de l'environnement affectant les sites d'accueil touristi- ques touchés par la pollution tellurique et marine.

La combinaison de tous ces facteurs négatifs pourraient entraîner un net recul de la fréquentation touristique compris entre 10 $\%$ et $20 \%$ pour l'ensemble de la Méditerranée (entre 10 et 15 millions de touristes en moins).

Dans ce cas, tous les foyers d'accueil méditerranéens seraient plus ou moins touchés, les plus affectés étant les périphéries méridionales et orientales sans effet de compensation suffisant, malgré le redéploiement d'une partie des touristes sur l'Europe méditerranéenne. Le scénario le plus noir pourrait être le résultat d'un cumul exceptionnel d'éléments négatifs: crises politiques majeures et durables dans les Balkans, au Moyen-Orient et en Afrique du Nord, crise socio-économique affectant directement le pouvoir d'achat d'une large partie des touristes européens (notamment en cas d'éclatement du SME). II faudrait plusieurs années de retour à la stabilité géopolitique et économique pour connaître unę nouvelle phase decroissance touristique. Al'heure actuelle, ce sont les troubles politiques en terre d'islam et dans 
FIGURE 3

Carte prospective
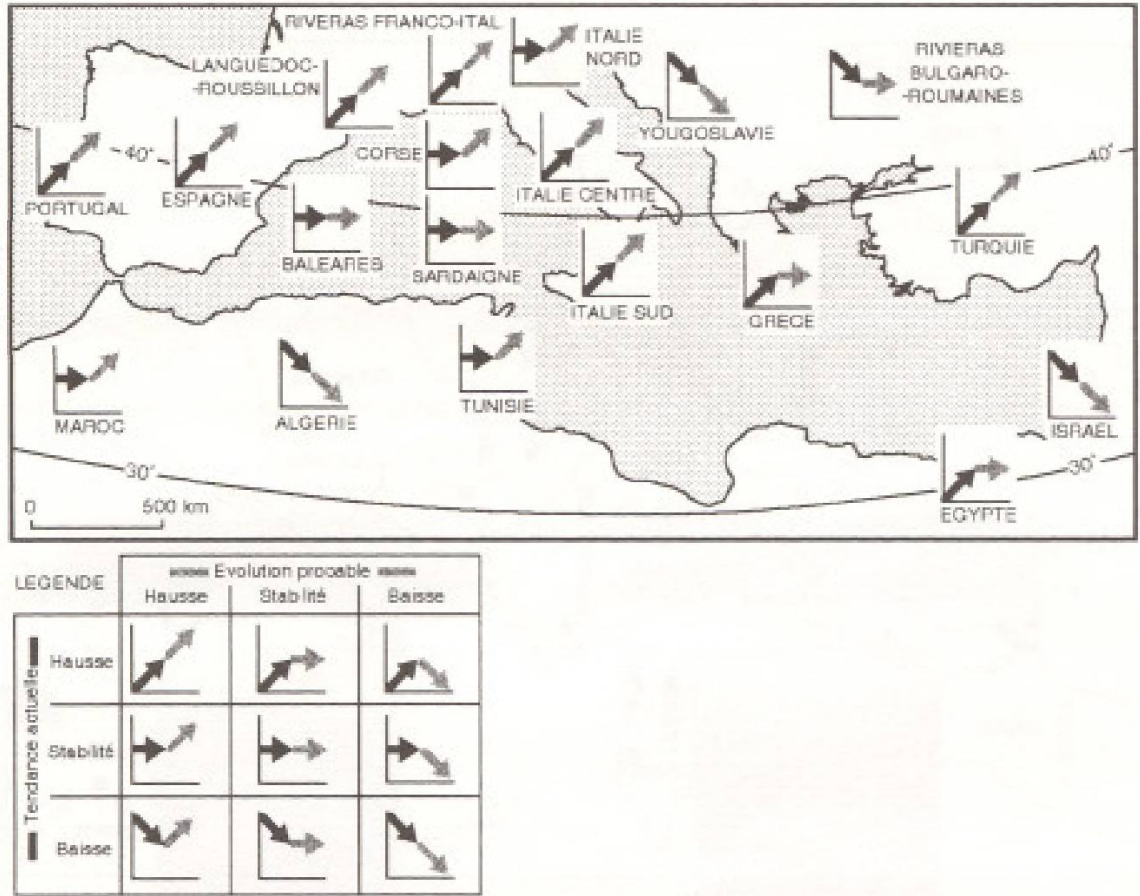

l'ex-Yougoslavie qui affaiblissent la fréquentation touristique à destination de la Méditerranée.

\section{Le scénario optimiste}

Depuis le début du tourismearistocratique et l'élan donné par le tourisme de masse, la Méditerranée n'a jamais connu qu'une longue croissance touristique à peine interrompue par les *accidents historiques liés aux grandes crises internationales (guertes mondiales, crisedes années 1930). Ni les crises régionales (Suez en 1956 ou la guerre du Golfe en 1991) et ni les récessions économiques de courte durée n'ont véritablement arrêtté l'expansion du tourisme en Méditerranée. C'est donc en se fondant sur la faible durabilité des secousses géopolitiques et socio-économiques qu'on peut envisager un scénario optimiste:

- fin rapide des conflits dans l'Europe balkanique et retour des touristes en Croatie, en Slovénie, en BosnieHerzégovine, en Roumanie et en Bulgarie;
- paix au Moyen-Orient et renouvean touristigue de la Syrie au Golfe Persique;

- renforcement socio-économique de la CEE et reprise massive des flux émetteurs touristiques vers l'ensemble des destinations méditerranéennes;

- enfin, amétioration sensible de l'état de Fenviromnement marin méditerranéen grâce aux effets positifs des plans internationaux (Plan Bleu) et des actions régionales et transfrontalières comme le Projet Ramage (défense de l'environnement marin entre $\mathrm{St}$ Raphaël, Monaco et Gểnes).

Ce scénario optimiste pourrait, éventuellement, confirmer les prévisions des années 1970-1980 annonçant une fortecroissance du tourisme en Méditerranée jusque dans la première décennie du XXIt siècle. Dans ce cas, tous les pays riverains continueraient à bénéficier, plus ou moins, d'un trend touristique positif-l'ex-Yougoslavie, l'Afrique du Nord et le Proche-Orient méditerranéen concurrençant de plus en plus les puissantes régions d'accueil touristiques de la Méditerranée nord-occidentale.

\section{Le scénario probable}

Entre les deux scénarios contradictoires, optimiste et pessimiste, il semble exister une troisième voie plus probable d'après les paramètres géopolitiques et géographiques auxquels semble de plus en plus sensible la fréquentation touristique en Méditerranée.

- L'instabilité géopolitique soulignée dans le scénario pessimiste devrait contribuer à l'affaiblissement des destinations touristiques nord-africaines et balkaniques.

- Les problèmes socio-économiquesde certains foyers émetteurs européens devraient prowoquer un recul sensible des flux émetteurs issus de certains pays (Grande-Bretagne, Italie, Espagne, Portugal, Grèce).

- En revanche, les touristes en provenancedel'Europe $*$ riche $»$ (pays scandinaves, Benelux, Allemagne, Autriche, Suisse, France), du Japon et del'Amérique du Nord devraient être plus nombreux au cours des prochaines années (les taux de change étant 
encoreplus favorables par rapport aux pays récepteurs méditerranéens).

- Enfin, l'effort d'équipement, quantitatifetqualitatif, en matière d'hôtellerieet d'infrastructures touristiques ne peut que favoriser la croissance du tourisme dans l'ensemble du bassin méditerranéen, à l'exception des zones conflictuelles (ex-Yougoslavie, Proche-Orient) ou instables(Algérie).

C'est donc un scénario réaliste, tablant sur une reprise de la croissance de la fréquentation touristique s'accompagnant d'un redéploiement à la fois vers le nord-ouest et le nord-est, qui apparaît comme le plus probable à l'horizon 2000. Situation cependant fragile à cause de deux paramètres difficiles à contrôler: les remous géopolitiques, d'une part, etla dégradation de l'environnement, d'autre part.

Par ailleurs, on peut aussi s'interroger sur l'éventuellesurcapacité d'accueil touristique due à la multiplication d'une offre soumise aux impératifs de l'économie d'échelle. La capacité moyenne de l'hébergement n'a pas cessé de croître partout en Méditerranée, commel'indiquent les divers plans régionaux de développement touristique. La stagnation du nombre de nuitées - en dehors même des reculs enregistrés depuis 1990 - un peu partout, à part exception (Turquie), est le résultat de la surcapacité de l'offre touristique par rapport au tassement de la demande. $\mathrm{La}$ concurrence des foyers d'accueil touristiques, intra et extraméditerranéens, pourrait aboutir à la remise en question d'objectifs et de choix touristiques pour tel ou tel secteur géographiquedu bassin méditerranéen. Le scénario réaliste incite doncà une plus grande prudence les décideurs, privés et publics, chargés du futur touristique en Méditerranée.

\section{Conclusion: de nouveaux atouts, de nouvelles contraintes}

La Méditerranée conserve de nombreux atouts pour rester le premier foyer d'accueil touristique mondial. Son potentiel artistique et son climat sont quasiment incontournables pour les nombreux touristes qui choisissent des destinations méditerranéennes. Cependant, il n'en demeure pas moins vrai que la forte pression touristique, sur l'espace et sur les hommes, renforcée par l'extension mal maittrisée de marinas, de complexes hôteliers et résidentiels, de golfs et de ports de plaisance, pourrait engendrer un phénomène de saturation ou de surcapacité refoulant une partie de la clientèle. La relative proximité géographique des foyers émetteurs européens et des foyers récepteurs méditerranéens n'est pas forcément garanti de la pérennité de la primauté touristique de la Méditerranée. De nos jours, les contraintes socio-économiques, géopolitiques et socio-psychologiques ne sont plus tout aussi favorables aux destinations touristiques méditerranéennes. Plus précisément, toute prospective sérieuse ne saurait faire l'impasse d'une analyse fouillée des externalités et des aménitér liées aux capacités quantitativer et qualitatives de laccueil, du service et de lanimation des diverses destinations méditerranéennes. C'est de cet ensemble de paramètres indissociables que va dépendre soit la stagnation, soit le recul soit la croissance du tourisme en Méditerranée. Pour notre part, nous pensons que l'évolution sera contrastée selon les régions et qu'il faudrait donc être plus sélectif dans les choix d'investissements et de promotion touristiques au cours des dix prochaines années. La prospective statistique et hôtelière traditionnelle devrait être complétée, voire précédée, d'une prospective spatiale à la fois plus globale et plus diversifiée. La Méditerranée pourrait donc ainsi devenir le premier laboratoire d'analyse spatiale des diachronies touristiques contemporaines.

\section{Notes explicatives}

(1) By OMT criteria, the tourism world is divided in five zones including mediterranean area in Europe, Middle-East and Africa.

(2) H. Kahn and A.J. Wiener, The Year 2000, Hudson Institute, 1967.

(3) Le Plan Bleu, dans le cadre du programme MABUNESCO de 1985, a fixé un certain nombre de priorités pour protéger l'environnement méditerranéenen fonction de prévisions démographiques et socio-économiques dont le tourisme n'est que l'une des composantes.

(4) La fiabilité de statistiques n'est que très relative. notamment en ce qui concerne le nombre global de touristes en Méditerranée. Contrairement à la plupart des organismes internationaux, il faudrait sans doute exclure des statistiques les régions non mediterranbennes, aussi bien en France que sur le continent africain.

(5) A. Edwards, Internutlonal Touriem Forecaste to 1995. The Economist Publications Ltd, London, 1985.

(6) J.P. Lozato-Giotart, Le Meroc, Editions Khartala, Paris, $1991,178 \mathrm{p}$.

(7) Cf. I'article de Catherine Ronchi, Maroc, toujours rien!, L'tcho tourlatique, Paris, no 2121, septembre 1991.

(6) G. Wackerman, Les liens touriatiqes d'un pace multinational de passige, le cas des pays rhénans, Colloque tourisme et coopúration internationale, Éditions Delta, Université de Nice 1977. pp. 63-88.

(9) W. Pahr, Lo tendenze del turiemo internazionale, Rassegna di studi turistici, ANIEST, a cura di Alberto Sessa, Casa Editrice Agnesotti, Roma, no 1, 1987, pp. 15-66. 